\title{
A candidate tidal disruption event in the Galaxy cluster Abell 3571
}

\author{
N. Cappelluti ${ }^{1,2}$, M. Ajello ${ }^{3,4}$, P. Rebusco ${ }^{5}$, S. Komossa ${ }^{1}$, A. Bongiorno ${ }^{1}$, C. Clemens ${ }^{1}$, M. Salvato ${ }^{6}$, P. Esquej ${ }^{7,1}$, \\ T. Aldcroft ${ }^{8}$, J. Greiner ${ }^{1}$, and H. Quintana ${ }^{9}$ \\ 1 Max-Planck-Institute für Extraterrestrische Physik, Postfach 1312, 85741, Garching bei München, Germany \\ e-mail: cap@mpe.mpg.de \\ 2 University of Maryland, Baltimore County, 1000 Hilltop Circle, Baltimore, MD 21250, USA \\ 3 SLAC National Accelerator Laboratory, 2575 Sand Hill Road, Menlo Park, CA 94025, USA \\ 4 KIPAC, 2575 Sand Hill Road, Menlo Park, CA 94025, USA \\ 5 Kavli Institute for Astrophysics and Space Research, MIT, Cambridge, MA 02139, USA \\ ${ }^{6}$ California Institute of Technology, 105-24 Robinson, 1200 East California Boulevard, Pasadena, CA 91125, USA \\ 7 Dept. of Physics and Astronomy, Leicester University, Leicester LE1 7RH, UK \\ 8 Harvard-Smithsonian Center for Astrophysics, 60 Garden St, Cambridge, MA 02138, USA \\ 9 Department of Astronomy and Astrophysics, Pontificia Universidad Catolica de Chile. Casilla 306, Santiago 22, Santiago, Chile
}

Received 5 December 2008 / Accepted 18 January 2009

\section{ABSTRACT}

Context. Tidal disruption events are possible sources of temporary nuclear activity in galactic nuclei and can be considered as good indicators of the existence of supermassive black holes in the centers of galaxies.

Aims. A new X-ray source has been detected serendipitously with ROSAT in a PSPC pointed observation of the galaxy cluster A3571. Given the strong flux decay of the object in subsequent detections, the tidal disruption scenario is investigated as a possible explanation of the event.

Methods. We followed the evolution of the X-ray transient with ROSAT, XMM-Newton and Chandra for a total period of $\sim 13$ years. We also obtained 7-band optical/NIR photometry with GROND at the ESO/MPI $2.2 \mathrm{~m}$ telescope.

Results. We report a very large decay of the X-ray flux of the ROSAT source identified with the galaxy LEDA 095953, a member of the cluster Abell 3571. We measured a maximum 0.3-2.4 keV luminosity $\log \left(L_{\mathrm{X}}\right)=42.8 \mathrm{erg} \mathrm{s}^{-1}$. The high state of the source lasted at least $150 \mathrm{ks}$; afterwards $L_{\mathrm{X}}$ declined as $\sim t^{-2}$. The spectrum of the brightest epoch is consistent with a black body with temperature $k T \sim 0.12 \mathrm{keV}$.

Conclusions. The total energy released by this event in $10 \mathrm{yr}$ was estimated to be $\Delta E>2 \times 10^{50} \mathrm{erg}$. We interpret this event as a tidal disruption of a solar type star by the central supermassive black hole (i.e. $\sim 10^{7} M_{\odot}$ ) of the galaxy.

Key words. X-rays: galaxies - galaxies: clusters: individual: Abell 3571 - galaxies: nuclei - X-rays: bursts - galaxies: active

\section{Introduction}

There is growing evidence that most galaxies with a bulge host a supermassive black hole $(\mathrm{BH})$ in their center (Kormendy \& Richstone 1995; Magorrian et al. 1998). The most recent theoretical and observational developments in the field of galaxy evolution suggest that during their life, galaxies experience one or more phases where the $\mathrm{BH}$ is active and powered by accretion. One of the effects of this phenomenon are the so called active galactic nuclei or quasars where accretion occurs on time scales longer than $10^{6-7} \mathrm{yr}$. It is widely accepted that during this period the $\mathrm{BH}$ is fed by an accretion disk. On the other hand, during the non-active phase of the galaxy life the BHs can be briefly powered by stars tidally disrupted during close encounters with the BHs (e.g. Frank \& Rees 1976). Recent studies (see e.g. Wang \& Merritt 2004; Esquej et al. 2008; Donley et al. 2002) pointed out that these events happen with a rate of $\sim 10^{-4}-10^{-5}$ galaxy $^{-1}$ year $^{-1}$. These phenomena can generate strong flares in the UV-X-ray band as the result of the onset of an accretion disk. The X-ray/UV luminosity of the galaxy is therefore expected to grow to values close to those of AGNs (i.e. $>10^{42} \mathrm{erg} \mathrm{s}^{-1}$ ). If the star is Sun-like, tidal disruption can only occur if the mass of the BHs does not exceed $\sim 10^{8} M_{\odot}$ since, in this case the tidal radius would typically fall within the Schwarzschild radius and the star is swallowed as a whole.
In the case of rotating $\mathrm{BHs}$, the tidal disruption also can occur for higher BH masses if the star approaches from a favorable direction (Beloborodov et al. 1992). However atmosphere stripping of giant stars is possible also for larger $\mathrm{BH}$ masses. When assuming a black body radiating at the Eddington luminosity, the temperature of the emitting region calculated at the last stable orbit ( $3 R_{\mathrm{S}}$, where $R_{\mathrm{S}}$ is the Schwarzschild radius) is $\sim 30-300 \mathrm{eV}$ for a $10^{6-7} M_{\odot}$ black hole. After the disruption, the mass accretion rate, and therefore the bolometric luminosity, may decay approximately as $t^{-5 / 3}$ (Rees 1990; Evans \& Kochanek 1989), even though deviations from this law are expected (see e.g. Lodato et al. 2008). 7 candidate tidal disruption events have been detected in the X-ray by the ROSAT all-sky survey and in the XMM-Newton slew survey (see e.g. Komossa \& Bade 1999; Greiner et al. 2000; Grupe et al. 1999; Komossa et al. 2004; Esquej et al. 2007). Candidate tidal disruption have been observed also in the Optical/UV (e.g. Renzini et al. 1995; Gezari et al. 2008). The X-ray events show strong X-ray variability (i.e. more than a factor of 100), $0.3-2.4 \mathrm{keV}$ luminosities typical of AGN and quasars (i.e. $>10^{42} \mathrm{erg} \mathrm{s}^{-1}$ ) declining as $\sim t^{-5 / 3}$, ultrasoft (i.e. $\sim 0.1 \mathrm{keV}$ ) X-ray spectra and the absence of Seyfert activity in ground-based optical spectra (see Komossa 2002, for a review). In this letter we report the serendipitous detection of a very large decay in the X-ray flux of the galaxy LEDA 095953 at $z=0.0366$ (Quintana \& de Souza 1993) in 

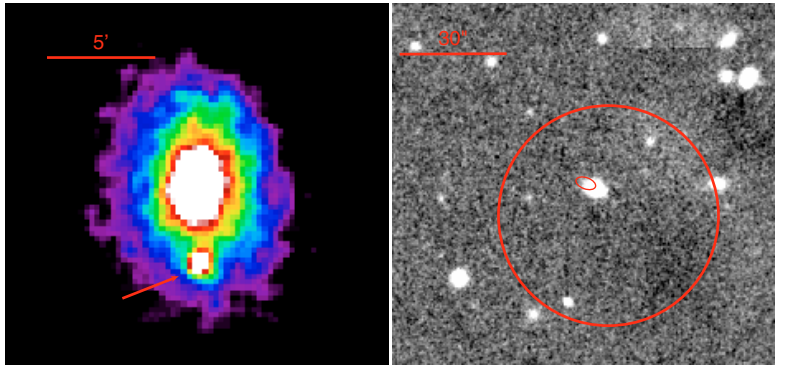

Fig. 1. Left panel: the ROSAT-PSPC 0.3-2.4 keV colour coded image of the field of A3571. The X-ray transient is indicated with the arrow. Right panel: GROND image in the $K_{\mathrm{S}}$-band of the region of the X-ray transient, the red circle represents the ROSAT-PSPC error-box and the red ellipsoid is the Chandra confidence region.

Table 1. The log of the X-ray and optical/NIR observation of the field of Abell 3571 used for this work.

\begin{tabular}{cccc}
\hline \hline Instrument & $\begin{array}{c}\text { Date } \\
\text { dd-mm-yyyy }\end{array}$ & Band & $\begin{array}{c}\text { Exposure } \\
\mathrm{s}\end{array}$ \\
\hline ROSAT-PSPC & $12-08-1992$ & $0.3-2.4 \mathrm{keV}$ & 5800 \\
ROSAT-HRI & $05-08-1994$ & $0.3-2.4 \mathrm{keV}$ & 19200 \\
XMM-Newton-EPIC-PN & $29-07-2002$ & $0.3-2.4 \mathrm{keV}$ & 24200 \\
Chandra-ACIS-S & $31-07-2003$ & $0.3-2.4 \mathrm{keV}$ & 33900 \\
ESO/MPI-GROND & $12-08-2008$ & $g, r^{\prime}, i^{\prime}, z^{\prime}$ & 458 \\
ESO/MPI-GROND & $12-08-2008$ & $J, H, K$ & 480 \\
\hline
\end{tabular}

the field of view of the galaxy cluster Abell 3571 using ROSAT, XMM-Newton and Chandra archival X-ray data and GROND optical/NIR follow-up.

\section{Observations and data processing}

During the soft X-ray follow-up of the Swift-BAT, hard X-ray selected sample of galaxy clusters (Ajello et al. 2008), we serendipitously discovered a bright source in a ROSAT-PSPC image of Abell 3571 (see Fig. 1) that apparently disappeared in subsequent observations with XMM-Newton and Chandra. The field of Abell 3571 has been observed in X-rays in pointed mode with the ROSAT-PSPC/HRI detectors, XMM-Newton EPIC, Chandra ACIS-S and during the ROSAT all-sky survey. The ROSAT data were processed using the standard MIDASEXSAS software version 03SEPpl1, while XMM-Newton and Chandra data were processed using XMM-SAS version 7.0 and CIAO 4.0. We also performed an optical/NIR follow-up of the field in the $g^{\prime}, r, i^{\prime}, z^{\prime}$ bands and $J, H, K_{\mathrm{S}}$ bands, by using GROND (Greiner et al. 2008), mounted at the $2.2 \mathrm{~m} \mathrm{ESO/MPI}$ telescope at La Silla observatory (Chile). In Table 1 we list the $\log$ of the observations of the field of Abell 3571 relevant for this work.

\subsection{Data analysis}

ROSAT-PSPC: using the ROSAT-PSCP $0.3-2.4 \mathrm{keV}$ energy band image and exposure map available in the archive, we created a background map, by first running a sliding cell source detection with a signal-to-noise ratio threshold of 4, excising the detected sources from the image and fittingthe residual data with a spline model. With the background map described above and using the exposure map we run a maximun likelihood, PSF-fitting, source detection. The bright, eye-detected, source mentioned in the previous section has been detected with a

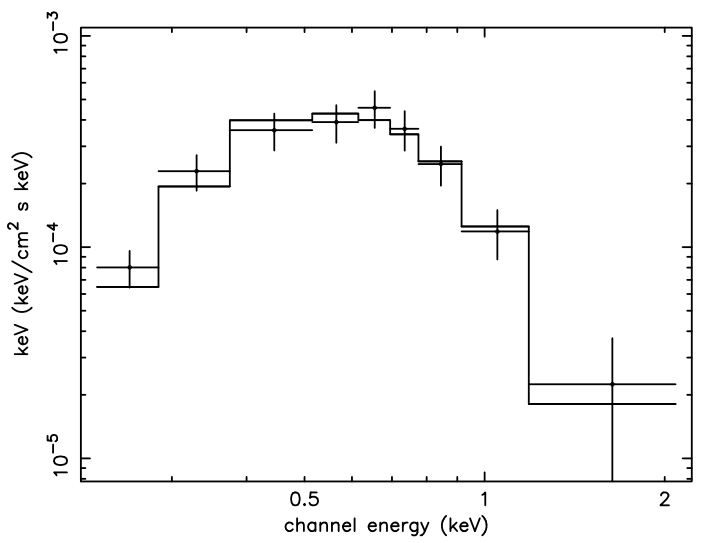

Fig. 2. The ROSAT-PSPC unfolded spectrum of the source TDXFJ134730.3-325451 (crosses) and the best fit blackbody model (continuous line).

significance $>10 \sigma$ with coordinates of the centroid $13^{\mathrm{h}} 47^{\mathrm{m}} 29.8^{\mathrm{s}}$ $-32^{\circ} 55^{\prime} 00^{\prime \prime}$ (J2000) with an error box of $\sim 25^{\prime \prime}$. Considering that the source is embedded in the X-ray emission of the galaxy cluster Abell 3571, the photometric properties have been evaluated using aperture photometry instead of using the estimate of the detection software. We extracted the source counts in a circular region with a radius corresponding to the $90 \%$ Encircled Energy Radius (EER) around the centroid estimated by the maximum likelyhood fit. Background counts have been estimated in a ring around the source with an inner radius 1.5 times the 90\% EER and outer radius 2 times the $90 \%$ ERR. As a result we obtained $305 \pm 27$ net source counts corresponding to a count-rate of $(5.2 \pm 0.4) \times 10^{-2} \mathrm{~s}^{-1}$. PSF fitting estimated a flux $\sim 30 \%$ lower as a result of a likely overestimate of the background. In the same regions we also extracted source and background lightcurves and spectra. All the epochs but one have flux consistent with a mean count-rate of $\sim 5.2 \times 10^{-2} \mathrm{~s}^{-1}$. Note that the observation was split in two parts separated by $\sim 150 \mathrm{ks}$. This indicates that no strong variability is present on such a time scale. However in one time bin the count-rate reached $(8.3 \pm 1.0) \times 10^{-2} \mathrm{~s}^{-1}$. This flux is $\sim 3 \sigma$ above the mean and could indicate a small flare during the event.

The unfolded spectrum of the source is shown in Fig. 2. We performed a $\chi^{2}$ fit to the spectrum using a black-body model plus cold absorption. We kept as free parameters the temperature $k T$, the columns density $N_{\mathrm{H}}$ and the normalization $k$. As a result we obtained a temperature $k T=120 \pm 16 \mathrm{eV}$ and $N_{\mathrm{H}}=$ $3.93_{-1.24}^{+1.43} \times 10^{20} \mathrm{~cm}^{-2}$ and $\chi^{2} / v=1.05 / 4.00$. This corresponds to a $0.3-2.4 \mathrm{keV}$ flux of $7.65 \pm 0.92 \times 10^{-13} \mathrm{erg} \mathrm{cm}^{-2} \mathrm{~s}^{-1}$. Assuming no spectral changes during the observation, the maximum $0.3-2.4 \mathrm{keV}$ flux emitted by the source is $1.16 \pm 0.13 \times$ $10^{-12} \mathrm{erg} \mathrm{cm}^{-2} \mathrm{~s}^{-1}$. Note that the column density is consistent within $1 \sigma$ with the Galactic value predicted by Dickey \& Lockman (1990) (i.e. $N_{\mathrm{H}}=3.7 \times 10^{20} \mathrm{~cm}^{-2}$ ) suggesting an extragalactic origin of the source. We also tested other spectral models, like a power-law plus absorption, without improving the quality of the fit by having $\Gamma \sim 6.5$, and $N_{\mathrm{H}} \sim 3 \times 10^{21} \mathrm{~cm}^{-2}$ as best fit parameters and $\chi^{2} / v=5.30 / 4.00$.

We also performed aperture photometry on ROSAT all sky survey images at the source location and we determined a $1 \sigma$ upper-limit (see next section) on the count-rate of $0.44 \mathrm{~s}^{-1}$ corresponding to a flux of $\sim 2.2 \times 10^{-12} \mathrm{erg} \mathrm{cm}^{-2} \mathrm{~s}^{-1}$ in the $0.3-2.4 \mathrm{keV}$ band assuming as a spectral model a black body with $k T=0.12 \mathrm{keV}$. 
ROSAT-HRI: the source is visually observable also in the HRI observation though much fainter than in the PSPC image. Considering the highest spatial resolution of the HRI detector we performed a source detection to constrain the source position better than in the PSPC pointing. Unfortunately because of the faintness of the source and the difficulty in modeling the high background due to the extended X-ray emission of the cluster, the source has been detected with a significance of $\sim 3-4 \sigma$, but as an extended source. We therefore performed aperture photometry around the ROSAT-PSPC centroid. By adopting the same procedure used for the PSPC data, we measured $38 \pm 9$ source counts corresponding to a count rate of $(1.97 \pm 0.45) \times 10^{-3} \mathrm{~s}^{-1}$. By assuming no spectral change between the ROSAT-PSPC and HRI observation (i.e. 2 years) we estimated a $0.3-2.4 \mathrm{keV}$ flux of $1.53 \pm 0.4 \times 10^{-13} \mathrm{erg} \mathrm{cm}^{-2} \mathrm{~s}^{-1}$.

XMM-Newton: a visual inspection of the EPIC-PN image does not show any relevant signature of a X-ray source in the ROSAT error box. We run a maximum likelihood source detection in that region but no sources were detected with significance $>3 \sigma$. We therefore estimated the $3 \sigma$ upper-limit of the source count at the source location by using the prescriptions of Narsky (2000). Given $M$ counts actually observed in a region of $30^{\prime \prime}$ (Cappelluti et al. 2009), and $B$ background counts, the $1 \sigma$ upper limit is defined as the number of counts $X$ that gives the probability to observe $M$ (or fewer) counts equal to the formal 68.3\% Gaussian probability: $P(\leq M, X+B)=P_{\text {Gauss }}(68.3 \%)$. Assuming Poissonian statistics this equation becomes: $P_{\text {Gauss }}=$ $\mathrm{e}^{-(X+B)} \sum_{i=0}^{M} \frac{(X+B)^{i}}{i !}$. By iteratively solving the previous equation in the case of ${ }^{i} P_{\text {Gauss }}=0.997$, we obtained the $3 \sigma$ upper limit $X=167$ counts. This corresponds to a count-rate of $8.8 \times$ $10^{-3} \mathrm{~s}^{-1}$ and to a flux of $1.45 \times 10^{-14} \mathrm{erg} \mathrm{cm}^{-2} \mathrm{~s}^{-11}$.

Chandra: using the task wavdetect, included in the CIAO software, we performed a source detection on the ACIS-S4 Chip where the source is observable. The analysis was performed in the $0.3-2.4 \mathrm{keV}$ energy band. We ran wavdetect using an exposure map estimated at $1 \mathrm{keV}$ with a threshold of $10^{-6}$, corresponding to a maximum of 1 spurious detection expected per ACIS-chip. As a result, in the ROSAT PSPC error box we detected one source with $9.5 \pm 3.74$ counts corresponding to a count-rate of $2.89 \times 10^{-4} \mathrm{~s}^{-1}$. The new source coordinates are $\alpha=13^{\mathrm{h}} 47^{\mathrm{m}} 30.33^{\mathrm{s}}, \delta=-32^{\circ} 54^{\prime} 50.63^{\prime \prime}$. At the source position the estimated $\sigma$ positional error is $\sim 2.5^{\prime \prime}$. We also ran a source detection in the $2-7 \mathrm{keV}$ band without detecting a source. We also estimated the flux of the source by folding into XSPEC the Chandra response matrices and assuming as the spectral model the ROSAT-PSPC best fit and obtained a $0.3-2.4 \mathrm{keV}$ flux of $3.43 \pm 1.35 \times 10^{-15} \mathrm{erg} \mathrm{cm}^{-2} \mathrm{~s}^{-1}$. Note that the source centroid lies about $5^{\prime \prime}$ from the chip edge, therefore the estimate of the background could be affected by features near this edge. According to its coordinate, the X-ray source has been named TDXFJ134730.3-325451.

GROND: in Fig. 1 we show the GROND $K$-band image of the area covered by the ROSAT-PSPC error box. As one can notice, the Chandra ellipsoid is placed right on the disk of a bright galaxy with an offset of $\sim 3^{\prime \prime}$ from the optical centroid of the

\footnotetext{
1 The flux was derived from the count-rate with the PIMMS software by assuming as the spectrum the ROSAT-PSPC best fit. http:// heasarc . nasa.gov/Tools/w3pimms.html
}

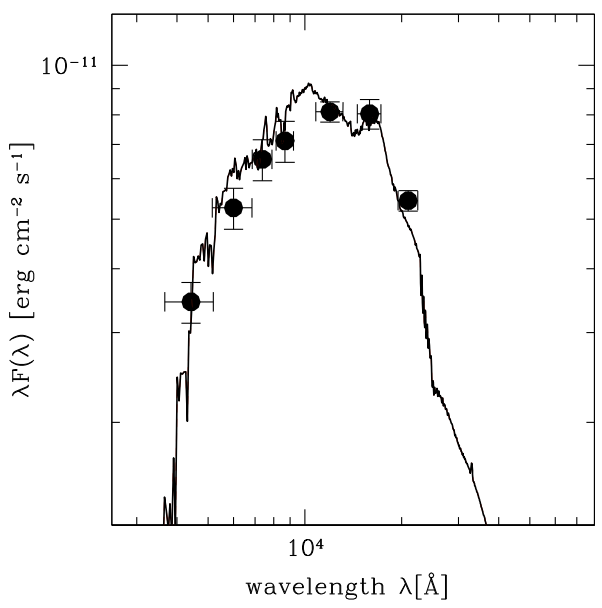

Fig. 3. The SED of the galaxy LEDA 095953 in the $g^{\prime}, r^{\prime}, i^{\prime}, z^{\prime}, H, J$ and $K_{\mathrm{S}}$ bands obtained with GROND over the best fit SED model of a S0 galaxy (solid line).

galaxy. Note that the GROND images have an astrometric uncertainty of $0.5^{\prime \prime}$. We searched the NED catalog and the galaxy was previously known as LEDA 095953, a member of the cluster Abell 3571 at $z=0.0366$ (Quintana \& de Souza 1993). We determined the photometric properties of the source and obtained the following $\mathrm{AB}$ magnitudes: 17.05 $\pm 0.10,16.26 \pm$ $0.10,15.80 \pm 0.10,15.54 \pm 0.10,15.04 \pm 0.02,14.75 \pm 0.07$ and $14.87 \pm 0.04$ in the $g^{\prime}, r^{\prime}, i^{\prime}, z^{\prime}, J, H, K_{\mathrm{S}}$ bands, respectively. These magnitudes are calculated by using SA105-815 and 2MASS stars as a reference and corrected for the expected foreground extinction of $E_{B-V}=0.054$ (Schlegel et al. 1998). We examined the global spectral energy distribution (SED) in Fig. 3 of this object using a tool based on a chi-squared minimization method that allows us to fit the observed fluxes with a combination of AGN and galaxy emission, also allowing for the possibility of extinction of the AGN flux. From this analysis we found that the model fit that better describes the SED of this object is a normal S0 galaxy with no significant contribution due to the presence of an AGN.

\section{Long term light curve}

The X-ray flux of TDXFJ134730.3-325451 has been followed for 13 years and its lightcurve is presented in Fig. 4. Remarkably, it shows an amplitude of the variability of a factor $>650$. We estimated the maximum $0.3-2.4 \mathrm{keV}$ luminosity ${ }^{2}$ as $\log \left(L_{X}\right)=$ $42.83 \mathrm{erg} \mathrm{s}^{-1}$. By excluding the upper limits, we fitted the long term lightcurve with a power-law model in the form: $L_{X}(t)=$ $k *\left(\frac{t-t_{\mathrm{disr}}}{1 \mathrm{yr}}\right)^{-\alpha}$, where $k$ is the normalization, $t_{\mathrm{disr}}$ marks the time of the star's disruption and $\alpha$ is the slope of the decay. As a result we obtained $k \sim 7 \times 10^{42} \mathrm{erg} \mathrm{s}^{-1}, t_{\text {disr }} \sim 1991.6$ and $\alpha=2.2 \pm 0.5$. Because of the low number of data points, the uncertainties on the fit parameters are large. The value of the slope $\alpha$ is therefore marginally consistent with the predictions of variants of tidal disruption models (i.e. $\alpha \sim 5 / 3$, see e.g. Evans \& Kochanek 1989; Rees 1990). Note that according to the lightcurve, the source at the maximum could have been at 1-2 orders of magnitude brighter with respect to the time of the ROSAT-PSPC observation. If the source continues its decay, it would be detectable with Chandra for all the year 2009 and 2010, with moderately deep exposures.

\footnotetext{
2 The luminosity has been computed assuming a $\Lambda$-dominated universe with $\Omega_{\Lambda}=0.7, \Omega_{\mathrm{m}}=0.3$ and $H_{0}=72 \mathrm{~km} \mathrm{~s}^{-1} \mathrm{Mpc}^{-1}$.
} 


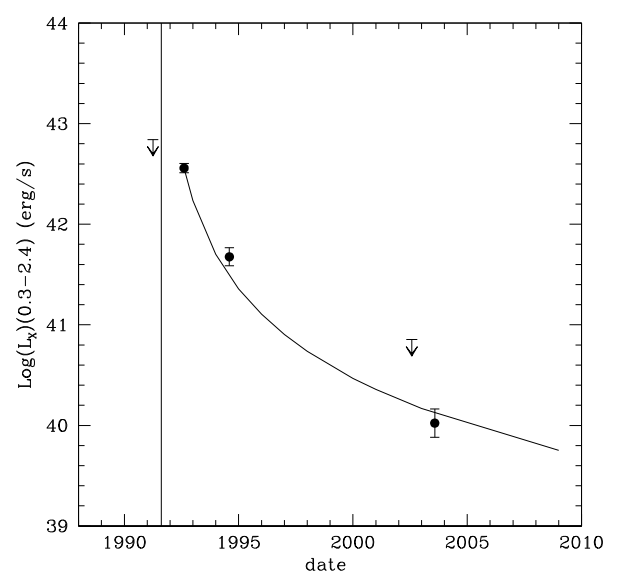

Fig. 4. The X-ray lightcurve of TDXFJ134730.3-325451. The solid curve represents the result of the power-law fit while the vertical line marks the expected begin of the flare.

\section{Discussion}

We serendipitously detected a factor $>650$ decay in the flux of an X-ray source in the rich galaxy cluster A3571. The flare most likely originated in the galaxy LEDA 095953 at $z=0.0366$. The most luminous epoch in the lightcurve reached $\log L_{X}=$ $42.83(0.3-2.4)$ and lasted for more than $150 \mathrm{ks}$, although higher luminosities at the time of the burst cannot be excluded. The spectrum of the flare is consistent with a black-body of temperature $k T \sim 0.12 \mathrm{keV}$ with no significant hard components. The optical/NIR SED of the host galaxy does not show any signature of AGN activity. In addition to this, the decay of the long term light curve is marginally consistent with $t^{-5 / 3}$, and thanks to the stringent RASS upper limit, we likely detected the source close to the burst. The X-ray luminosity and the variability of the source are therefore much higher than those of ULX, which have $L_{X}<10^{39-40} \mathrm{erg} \mathrm{s}^{-1}$ and flux variations typically of up to a factor of 2-3 (see e.g. Colbert \& Ptak 2002). Supernovae explosions can also produce X-ray flares, but these are either of very short duration (hours), or else if longer lasting they have much harder X-ray spectra and typically have $L_{\mathrm{X}}<10^{41} \mathrm{erg} \mathrm{s}^{-1}$ (see e.g. Immler \& Lewin 2003, for a review). Additionally novae and supersoft sources can produce bursts but with much shorter duration (Tanaka \& Shibazaki 1996). According to the event properties, we can safely exclude these kinds of stellar phenomena. However, such a strong variability could be observed in GRB afterglows or Hypernovae but with a much shorter duration of the outburst. The characteristics of the flare are therefore consistent with previous cases of candidate tidal disruption events (see e.g. Komossa et al. 2004; Esquej et al. 2008) and with theoretical predictions (Rees 1990; Lodato et al. 2008). The mass of the BH should be not much higher than $\sim 10^{8} M_{\odot}$ to disrupt a Sun-like star outside the Schwarzschild radius and to allow the onset of accretion. We then estimated the black hole mass from the $L_{K}-M_{\mathrm{BH}}$ relation determined by Marconi \& Hunt (2003) in the local Universe. If $L_{K}$ is the $K$-band luminosity of the bulge of the galaxy in solar units then $\log \left(M_{\mathrm{BH}} / M_{\odot}\right) \sim 8.08+1.21\left(L_{K_{\text {bulge }}} / L_{\odot}-10.9\right)$. Using an aperture radius of $\sim 4^{\prime \prime}$ (i.e. $\sim 2.5 \mathrm{kpc}$, at the galaxy distance) we estimated a $\log \left(L_{K_{\text {bulge }}}\right) \sim 10 L / L_{\odot}$ which corresponds to $\log \left(M_{\mathrm{BH}} / M_{\odot}\right) \sim 7$. The total energy released during the event can be estimated with $\Delta E_{\mathrm{X}}=\int_{t}^{\infty} L_{\mathrm{X}}(t) \mathrm{d} t$, where $L_{\mathrm{X}}(t)$ is the best fit to the light curve. By integrating over a period of $\sim 10 \mathrm{yr}$ after our first data point we obtained a total energy release of the order of $2 \times 10^{50} \mathrm{erg}$. If $\epsilon \sim 0.1$ is the typical efficiency of mass to energy conversion for accretion onto a $\mathrm{BH}$, then the total mass accreted during a 10 year period is $\Delta M \sim \Delta E_{\mathrm{X}} / \epsilon c^{2}$. This yields a total mass deposition of $1 \times 10^{-3} M_{\odot}$. This value is actually a lower limit, since we started the integration only at the start of the observations (note that the fitting function diverges at the time of the disruption). Moreover it is possible that the total emission is dominated by an unobserved EUV component (extrapolation of the single black body fit itself predicts a $7 \%$ higher luminosity), and the true accretion rate is much higher. According to the results of Li et al. (2002), for a black hole of $10^{7} M_{\odot}$, the flare occurs $\sim 0.07 \mathrm{yr}$ after the disruption. A $10 \mathrm{yr}$ integration of the lightcurve model from this epoch yields an estimate of the accreted mass of $\sim 0.03 M_{\odot}$. A numerical simulation by Ayal et al. (2000) showed that for a $1 M_{\odot}$ star disrupted by a $10^{6} M_{\odot} \mathrm{BH}$, only a fraction of the order of $\sim 10 \%$ of the initial mass is actually accreted. Our limits on the accreated mass are therefore consistent with the scenario of a disruption of a $1 M_{\odot}$ star by the BH. However a partial stellar disruption or an explosive disruption (Brassart \& Luminet 2008) cannot be ruled out, although disruption and accretion of a brown dwarf or of a planets is very unlikely. We note that since the tidal disruption rates are likely of the order of $10^{-4}-10^{-5}$ galaxy $^{-1} \mathrm{yr}^{-1}$, galaxy clusters are ideal laboratories to explore this phenomenon: by conservatively assuming 100 member galaxies per cluster (Reiprich \& Böhringer 2002), one should expect 1 tidal disruption event per cluster every 10-100 yrs. The eROSITA all-sky survey (Predehl et al. 2006) will detect several thousands of galaxy clusters and therefore a large number of these events.

Acknowledgements. The XMM-Newton, Chandra and ROSAT archival systems and the full GROND team are acknowledged. P.R. is supported by the Pappalardo fellowship at MIT. H.Q. thanks partial support from the FONDAP Centro de Astrofisica.

\section{References}

Ajello, M., et al. 2008 [arXiv:0809.0006]

Ayal, S., Livio, M., \& Piran, T. 2000, ApJ, 545, 772

Beloborodov, A. M., Illarionov, A. F., Ivanov, P. B., \& Polnarev, A. G. 1992, MNRAS, 259, 209

Brassart, M., \& Luminet, J.-P. 2008, A\&A, 481, 259

Cappelluti, N., et al. 2009 [arXiv:0901.2347]

Colbert, E. J. M., \& Ptak, A. F. 2002, ApJS, 143, 25

Dickey, J. M., \& Lockman, F. J. 1990, ARA\&A, 28, 215

Donley, J. L., Brandt, W. N., Eracleous, M., \& Boller, T. 2002, AJ, 124, 1308

Esquej, P., Saxton, R. D., Freyberg, M. J., et al. 2007, A\&A, 462, L49

Esquej, P., Saxton, R. D., Komossa, S., et al. 2008, A\&A, 489, 543

Evans, C. R., \& Kochanek, C. S. 1989, ApJ, 346, L13

Frank, J., \& Rees, M. J. 1976, MNRAS, 176, 633

Gezari, S., Basa, S., Martin, D. C., et al. 2008, ApJ, 676, 944

Greiner, J., Schwarz, R., Zharikov, S., \& Orio, M. 2000, A\&A, 362, L25

Greiner, J., Bornemann, W., Clemens, C., et al. 2008, PASP, 120, 405

Grupe, D., Thomas, H.-C., \& Leighly, K. M. 1999, A\&A, 350, L31

Immler, S., \& Lewin, W. H. G. 2003, SNe and GRBs, 598, 91

Kormendy, J., \& Richstone, D. 1995, ARA\&A, 33, 581

Komossa, S. 2002, Rev. Mod. Astron., 15, 27

Komossa, S., \& Bade, N. 1999, A\&A, 343, 775

Komossa, S., Halpern, J., Schartel, N., et al. 2004, ApJ, 603, L17

Li, L.-X., Narayan, R., \& Menou, K. 2002, ApJ, 576, 753

Lodato, G., King, A. R., \& Pringle, J. E. 2008 [arXiv: 0810 . 1288]

Magorrian, J., Tremaine, S., Richstone, D., et al. 1998, AJ, 115, 2285

Marconi, A., \& Hunt, L. K. 2003, ApJ, 589, L21

Narsky, I. 2000, Nucl. Inst. Meth. Phys. Res. A, 450, 444

Predehl, P., Hasinger, G., Böhringer, H., et al. 2006, Proc. SPIE, 6266

Quintana, H., \& de Souza, R. 1993, A\&AS, 101, 475

Rees, M. J. 1990, Science, 247, 817

Reiprich, T. H., \& Böhringer, H. 2002, ApJ, 567, 716

Renzini, A., Greggio, L., di Serego-Alighieri, S., et al. 1995, Nature, 378, 39

Tanaka, Y., \& Shibazaki, N. 1996, ARA\&A, 34, 607

Schlegel, D. J., Finkbeiner, D. P., \& Davis, M. 1998, ApJ, 500, 525

Wang, J., \& Merritt, D. 2004, ApJ, 600, 149 